MATEMATIC. UNA EXPERIENCIA DE AULA QUE INTEGRA LAS MATEMÁtICAS Y LAS TIC

\title{
MatemaTIC. Una experiencia de aula que integra las matemáticas y las $\mathrm{TIC}^{1}$
}

Matematic. A classroom experience that integrates mathematics and ICT

Matematic. A experiência em sala de aula que integra matemática e TIC

Recibido: mayo de 2013

Aceptado: agosto de 2013
Juan Manuel Zuluaga ${ }^{2}$

Franklin Eduardo Pérez ${ }^{3}$ Juan Diego Gómez ${ }^{4}$

\section{Resumen}

En este texto se presenta una experiencia de aula desarrollada con un grupo aproximado de 170 estudiantes del grado décimo del Instituto San Carlos (ISC). En el 2012 se propuso la apertura de un blog en la web, constituido por cuatro diferentes productos asociados a cuatro fases.

Palabras clave: Matemáticas; TIC; aprendizaje; didáctica; proyecto de aula; blog; recursos didácticos, recursos informáticos.

\section{Abstract}

This text presents a classroom experience developed with a group of approximately 170 tenth graders San Carlos Institute (CSI). In 2012 it was proposed to open a blog on the web, consists of four different products associated with four phases.

Keywords: Mathematics, ICT, learning, teaching, classroom project, blog, teaching resources, resources.

\section{Resumo}

Este texto apresenta uma experiência de sala de aula desenvolvida com um grupo de cerca de 170 décimo Instituto Carlos San alunos (CSI). Em 2012, foi proposto para abrir um blog na web, é composta por quatro produtos diferentes associados com quatro fases.

Palavras-chave: Matemática, TIC, aprendizagem, ensino, projeto de sala de aula, blog, recursos pedagógicos, recursos.

1 Artículo de Investigación.

2 Instituto San Carlos de la Salle. Colombia. Contacto: mmanuel@ayura.udea.edu.co

3 Secretaría de Educación de Medellín. Colombia. Contacto: talleresconclase@gmail.com

4 Instituto San Carlos de la Salle. Colombia. Contacto: diegogo21@gmail.com, 


\section{Objetivos}

1) Mostrar que las experiencias con las matemáticas puede ser mediadas por las TIC. 2) Mostrar que los procesos creativos y las matemáticas tienen nodos comunes. 3) Dinamizar las relaciones que tienen los estudiantes con las matemáticas, sus profesores y compañeros. 4) Propiciar ambientes investigativos en las aulas de clase de la media vocacional.

\section{Metodología}

La propuesta surgió bajo el deseo de mediar el aprendizaje de las matemáticas con experiencias y estrategias no convencionales. Los ejercicios previos buscaban hallar un equilibrio entre el goce y disfrute del ejercicio artístico-creativo, aplicación tecnológica y la rigurosidad que exigen las matemáticas, equilibrio que permitió generar espacios de investigación, elaboración de conceptos, solución de problemas, búsquedas, etc. Se planteó la construcción de un blog obligatorio por parejas o tríos de estudiantes; este soportaría, guardaría y recopilaría una serie de productos que los estudiantes construirían por trimestre. Las actividades fueron: 1. Creación de un blog y búsqueda de un concepto matemático. 2. Creación de un vídeo animado con la técnica stop motion. 3. Creación de un cortometraje. 4. Creación de un vídeo, tipo noticiero.

\section{Matematic favorece el desarrollo de pensa- mientos tales como}

Pensamiento Variacional. En la interpretación, análisis y manejo que el estudiante hace en sus trabajos, que en este caso particular son sobre conceptos trigonométricos.

Pensamiento Geométric. En el manejo y distribución de los espacios, escenarios, formas y figuras. Determinados para cada una de las actividades propuestas, este pensamiento se ve reflejado en el manejo propio de los conocimientos específicos de la trigonometría.

Pensamiento Métric. En el manejo y distribución de unidades de tiempo, recursos, etc.

\section{Estilos los de aprendizaje}

MatemaTIC considera los diferentes ritmos y estilos de aprendizaje en la medida en que los estudiantes pueden elegir diferentes alternativas, grupos de trabajo, fuentes, herramientas para alcanzar las metas propuestas.

Dado que cada estudiante cuenta con unas habilidades referidas a su ritmo y estilo de aprendizaje asume un rol particular en el proceso de construcción de cada producto.

\section{Resultados}

En esta experiencia de aula se obtuvieron como resultados todos los productos multimediales que los estudiantes construyeron y publicaron en sus blogs.

Como valor agregado se evidencia el placer y la satisfacción que los estudiantes expresaron frente a las actividades propuestas en el proceso y el cambio de actitud que los mismos presentaron frente a las matemáticas.

http://matematicisc.wix.com/matematic

http://www.youtube.com/watch?feature=player_embedded\&v=D_c2AEdL3oI http://pelodematematicas.blogspot.com/

http://mathmanuel.blogspot.com/

\section{Conclusiones}

1) A través de los productos obtenidos por los estudiantes se puede evidenciar una estrecha relación 
entre la tecnología, las matemáticas y su aprendizaje. 2) El impacto fue muy positivo para los estudiantes, quienes encontraron en esta propuesta una forma diferente de ver y de aprender las matemáticas. 3) Se dinamizaron las clases, en la medida en que se generaron espacios de interacción, discusión y argumentación de los diferentes blogs y conceptos abordados en los mismos. 4) Se promueve un espíritu investigativo y se muestra una imagen no lineal del conocimiento.

\section{Referencias}

Bohórquez, E. (2008). El blog como recurso educativo. EDUTEC, Revista Electrónica de Tecnología Educativa, 26, 1-10.

DE MATEMATICAS, M. L. C. A. (1998). Serie Lineamientos Curriculares. Bogotá Julio de.

Frabetti, C. (2009). Literatura y matemáticas. Uno: Revista de Didáctica de las Matemáticas, 13 (50), 42-46.

Gloria, A., \& Patiño, R. Maestros e intelectuales en la educación colombiana. En Memorias IV simposio Internacional Horizontes Humanos p. $51)$.
Jorba, J. \& Sanmartí, N. (1996) Enseñar, aprender y evaluar: un proceso de regulación continua: Propuestas didácticas para las áreas de ciencias de la naturaleza y matemáticas. Madrid: Ministerio de Educación Nacional

Osorio, M. (2012). Unidad 3: Formas de entender la tecnología en un proceso enseñanza-aprendizaje: Formación presencial mediada por la tecnología, formación combinada y formación virtual. En curso virtual Asesoría para el uso de las TIC en la Formación. Bogotá: SENA.

Pimm, D. (1990). El lenguaje matemático en el aula (Vol. 15). Ediciones Morata.

Ramírez Vega, A., \& Chacón Rivas, M. (2011, December). Math Bridge: una propuesta como apoyo en los procesos de enseñanza-aprendizaje de las matemáticas en Costa Rica (CO). En XIII Conferência Interamericana de Educação Matemática.

Uribe Calad, Julio. (1994) Matemáticas básicas y operativas. Susaeta Ediciones S.A. 